\title{
Delayed gamma-ray spectroscopy combined with active neutron interrogation for nuclear security and safeguards
}

\author{
Mitsuo Koizumi ${ }^{1}$ a, Fabiana Rossi ${ }^{1}$, Douglas C. Rodriguez ${ }^{1}$, Jun Takamine ${ }^{1}$, Michio Seya ${ }^{1}$, Tatjana Bogucarska ${ }^{2}$, \\ Jean-Michel Crochemore ${ }^{2}$, Giovanni Varasano ${ }^{2}$, Kamel Abbas ${ }^{2}$, Bent Pederson ${ }^{2}$, Masatoshi Kureta ${ }^{3}$ Jan Heyse $^{4}$, \\ Carlos Paradela ${ }^{4}$, Willy Mondelaers ${ }^{4}$, and Peter Schillebeeckx ${ }^{4}$ \\ 1 Integrated Support Center for Nuclear Security and Nuclear Nonproliferation, Japan Atomic Energy Agency, Shirakata Shirane 2-4, \\ Tokai, Naka, Ibaraki, Japan \\ 2 Nuclear Safety and Security, Joint Research Centre in, via Enrico Fermi 2749, 21027 Ispra (VA), Italy \\ 3 Nuclear Science and Engineering Center, Japan Atomic Energy Agency, Shirakata Shirane 2-4, Tokai, Naka, Ibaraki, Japan \\ ${ }^{4}$ Nuclear Safety and Security, Joint Research Centre, Retieseweg 111, 2440 Geel, Belgium
}

\begin{abstract}
For the purpose of nuclear security and safeguards, an active neutron interrogation non-destructive assay technique, Delayed Gamma-ray Spectroscopy (DGS), is under development. The technique of DGS uses the detection of decay $\gamma$ rays from fission products to determine ratios of fissile nuclides in a sample. A proper evaluation of such $\gamma$-ray spectra requires integration of nuclear data such as fission cross-sections, fission yields, half-lives, decay-chain patterns, and decay $\gamma$-ray yields. Preliminary DGS experiments with the Pulsed Neutron Interrogation Test Assembly, named PUNITA, of the European Commissions' Joint Research Center have been performed. Signals of delayed $\gamma$ ray from nuclear materials were successfully observed.
\end{abstract}

\section{Introduction}

Due to the global increase of applications using nuclear materials (NMs), the requirements of effective characterization methods is growing in nuclear security and safeguards. Mass verification of NMs with low radioactivity is generally performed using passive nondestructive analysis (NDA) techniques whereas destructive analysis (DA) techniques are applied for accurate analysis of nuclide composition. A sample with high radioactivity, however, has a difficulty in characterization by passive NDA methods. Active NDA techniques, which utilize interrogation particles (such as photons and neutrons) to induce nuclear reactions, are potentially applicable to extract information of NMs in a high radioactive sample. Such active interrogation methods could also be used for the detection of clandestine NM.

An active-neutron NDA system equipped with a D-T pulsed-neutron generator is currently under development [1] at the Japan Atomic Energy Agency (JAEA). Multiple techniques are expected to be introduced to the system: Differential Die Away Analysis (DDA) [2,3] for the determination of total fissile content, Delayed Gamma-ray Spectroscopy (DGS) [4-7] for the evaluation of relative mass ratio of fissile nuclides (i.e., ${ }^{235} \mathrm{U},{ }^{239} \mathrm{Pu}$, and ${ }^{241} \mathrm{Pu}$ ), Neutron Resonance Transmission Analysis (NRTA) [8] for quantification of $\mathrm{U}$ and $\mathrm{Pu}$ isotopes, and Prompt Gammaray Analysis (PGA) [8] for detection and quantification of specific nuclides. Technical developments of those methods are in progress under the collaboration with the European Commission's Joint Research Center (EC-JRC). This paper describes the DGS development program and

$\overline{\text { a e-mail: koizumi.mitsuo@jaea.go.jp }}$ experimental results of DGS carried out with the Pulsed Neutron Interrogation Test Assembly (PUNITA) $[9,10]$ of EC-JRC in Ispra.

\section{Nuclear data in DSG}

DGS measures decay $\gamma$ rays from fission products (FP). The observed $\gamma$-ray peak intensity ratios of a spectrum are used for determining the ratio of the original fissile nuclides. Utilization of high-energy (HE) delayed $\gamma$ rays from relatively short-lived isotopes are considered useful for avoiding the overlap of background $\gamma$ rays from longlived radioactivity in a sample $[6,7]$.

A proper evaluation of delayed $\gamma$-ray spectra requires integration of nuclear data that describes phenomena involved in DGS (see Table 1): (1) Neutrons introduced in an experimental apparatus scatter, disperse, and cause nuclear reactions. Secondly neutrons are produced by nuclear reactions (mostly fission) and delayed neutron emissions. (2) Radioactive nuclides are produced by the result of nuclear reaction (i.e., fission and capture reaction). The produced radioactive nuclides start sequential disintegration to be stable nuclides; partially, they capture a neutron. The population of each nuclide changes at the every moment of DGS process. (3) A $\gamma$-ray spectrum is formed in a measurement of decay $\gamma$ rays emitted from the produced radioactive nuclides in their disintegrating process.

Nuclear data required to reproduce the phenomena are reaction cross-sections (such as fission), fission yields (FY), half-lives, decay-chain patterns, and decay $\gamma$-ray yields. Monte Carlo simulations are, therefore, useful for analyzing a DGS spectrum, in which many processes are involved in.

(C) The Authors, published by EDP Sciences. This is an Open Access article distributed under the terms of the Creative Commons Attribution License 4.0 (http://creativecommons.org/licenses/by/4.0/). 
Table 1. Phenomena involved in DGS measurement.

\begin{tabular}{|l|}
\hline (1) neutron field generation \\
\hline interaction of neutron \\
$\quad$ - elastic and inelastic scattering \\
- capture* \\
$\quad$ - fission* \\
$\quad$ - reaction $((\mathrm{n}, 2 \mathrm{n}), \text { etc. })^{*}$ \\
delayed neutron emission \\
\hline (2) production of radioactivity \\
\hline interaction of neutron \\
$\quad$ items with $*$ listed above \\
disintegration of radioactive nuclei \\
\hline$(3) \gamma$-ray measurement \\
\hline$\gamma$-ray emission in disintegration \\
photon reaction with materials \\
\hline
\end{tabular}

Examination and confirmation of nuclear data are, therefore, important in the development of DGS method as well as technical development. The FY distribution in evaluated nuclear databases are only for the incident energy of thermal $(0.0253 \mathrm{eV})$, fast $(500 \mathrm{keV})$, and $14 \mathrm{MeV}$. Information between those energies is essentially lost, while neutrons of wide range of energy participate in DGS. Those of the first resonances at about $0.3 \mathrm{eV}$ of fissile nuclides have to be investigated, because the fission process should pass through a different nuclear resonance state from that with thermal neutrons. For that purpose, the double energy mass measurements of fissile nuclides [14] at GELINA [15] of EC-JRC Geel are under consideration. In addition, the decay branching ratio and $\gamma$-ray yield, as well as FY, are critical for DGS. Confirmation, revision, and improvement of the nuclear data values and of uncertainty are probably required.

\section{DGS experiments with PUNITA}

DGS experiments were carried out with PUNITA $[9,10]$, which consists of a large graphite inner box and a high density polyethylene (HDPE) outer box. The inside of the box is a sample cavity of $50 \mathrm{~cm}$ in width $(\mathrm{W}), 50 \mathrm{~cm}$ in length (L), and $80 \mathrm{~cm}$ in height $(\mathrm{H})$. A standard pulsed 14-MeV D-T neutron generator (Thermo Fisher Scientific Corp., A-211) is placed in it. The specification value of the generator is $2 \times 10^{8} \mathrm{n} / \mathrm{s}$ neutron emission. Around the generator, tungsten rings with a thickness of $4.5 \mathrm{~cm}$ is used for slowing down the neutrons and for neutron multiplication by the $(\mathrm{n}, 2 \mathrm{n})$ reactions [10].

In DGS experiments, one of the cavity walls is partially open for sample transportation between the cavity and a $\gamma$-ray detector system, which is about $1 \mathrm{~m}$ away from PUNITA. This open channel allows some escape of neutrons to decrease the neutron flux in the cavity. In addition, high-energy neutrons (more than $500 \mathrm{keV}$ ) open new channels of fission reaction, and make the FY pattern different. This makes the DGS analysis complicated. Therefore, neutron field is desired to be as well thermalized and high flux as possible.

By using MCNP [11], models are investigated to determine the effect of additional moderator and reflector material to increase the thermal neutron flux at the sample irradiation point. Figure 1 shows an optimized geometry. In this calculation, a carbon block of $26 \mathrm{~cm}$ (W), $26 \mathrm{~cm}$ $(\mathrm{L})$, and $38 \mathrm{~cm}(\mathrm{H})$ with an 18-cm-square tunnel for sample

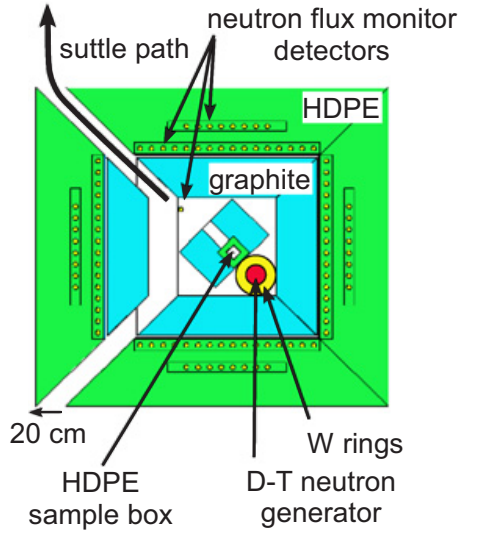

Figure 1. Horizontal cross-sectional geometry for a simulation of PUNITA. Additional moderators and reflectors were installed in the PUNITA cavity. The HDPE sample box is placed as near as possible.

Table 2. Experimental and calculated neutron flux normalized by $10^{8} \mathrm{n} / \mathrm{s}$ neutron emission from a neutron generator. The unit of the flux is $\times 10^{3} \mathrm{n} / \mathrm{cm}^{2} / \mathrm{s}$.

\begin{tabular}{|l|c|cc|cc|}
\hline geometry & (a) & \multicolumn{2}{|c|}{ (b) } & \multicolumn{2}{c|}{$\left(\mathrm{b}^{\prime}\right)$} \\
\hline & sim. & sim. & expt. [10] & sim. & expt. [9] \\
\hline thermal & 150 & 8.4 & 10 & 4.8 & 6.0 \\
14-MeV & 7.7 & 2.5 & - & 5.4 & 6.2 \\
\hline
\end{tabular}

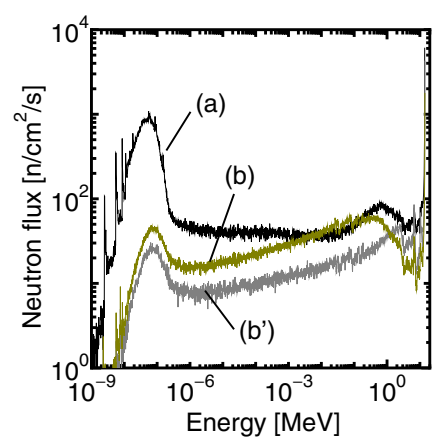

Figure 2. Calculated neutron energy spectra. (a) The geometry of Fig. 1 is used. (b) Neutron spectrum at the center of the cavity with no additional moderators and reflectors, and the shuttle path is closed. ( $b^{\prime}$ ) Same as (b) but the W rings is also removed.

transportation was installed. The sample volume is placed close to the neutron source point, and is surrounded by a $3 \mathrm{~cm}$ thick HDPE filter. The carbon block works for reflecting neutrons (i.e., preventing them from going away) and the HDPE box for slowing down them.

The calculated neutron energy spectrum with an additional moderator and reflector is given by plot (a) in Fig. 2, where (b) are that at the center of the cavity without the additional moderator and reflector, and with the cavity wall closed; $\left(b^{\prime}\right)$ is that obtained with further removal of the $\mathrm{W}$ rings. Table 2 shows experimental and calculated neutron fluxes normalized by $10^{8} \mathrm{n} / \mathrm{s}$ neutron emission, where the thermal flux is the integral less than $0.5 \mathrm{eV}$, and the $14-\mathrm{MeV}$ flux is the integral from $13 \mathrm{MeV}$ to $15 \mathrm{MeV}$. The simulation results (b) and $\left(\mathrm{b}^{\prime}\right)$ shows that the $\mathrm{W}$ rings increase neutrons of thermal and moderate energy $(<1 \mathrm{MeV})$ regions. The geometry (a) increases thermal flux much from that of (b). 


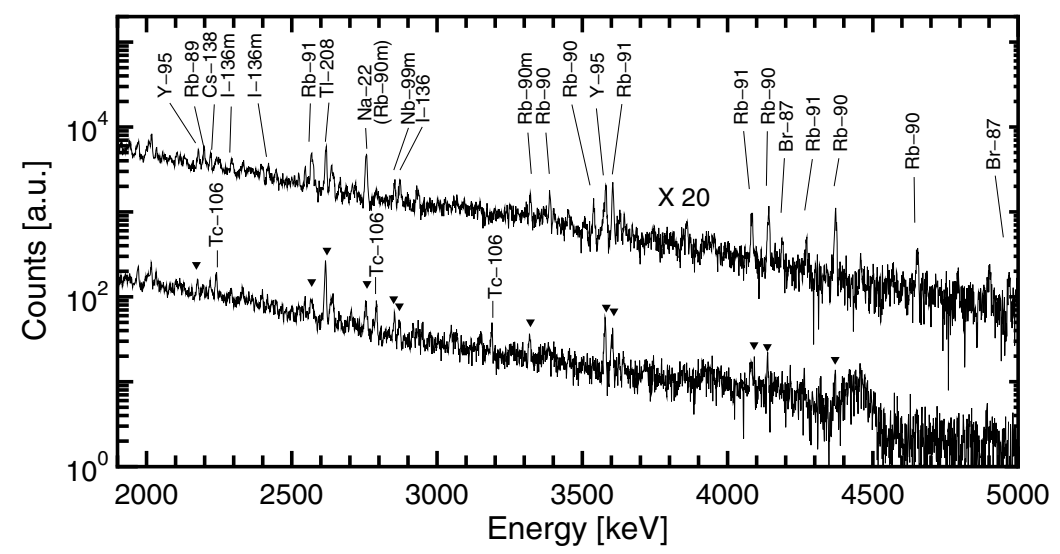

Figure 3. $\gamma$-ray Spectra of CBNM-446 (upper) and a Pu (bottom) sample, where the upper spectrum is multiplied by 20 for readers' sake. The triangle marks indicate that the same peak identification of the upper spectrum.

Because there was a difficulty in constructing the same geometry as Fig. 1, available HDPE and carbon blocks were piled up so as to reproduce the similar structure, in experiments.

Two HP Ge detectors were used for measurement: CANBERRA GR-2520 (P-type 25\%), and ITECH Instruments NIGC 50220 (N-type 50\%). The two outputs of each HP Ge detector were sent to two different data acquisition (DAQ) systems. One was a conventional NIM based system consisting of spectroscopy amplifiers (Ortec 672 and 673) and a dual-input multichannel analyzer system (Otrec Aspec-927). The other was a waveform digitizer (CAEN V1730) equipped with pulse height analyzing firmware (CAEN DPP-PHA). This digitizer analyses an input waveform to evaluate its pulse height, which is then stored event-by-event manner (list mode measurement) with a time stamp.

DGS measurements repeated the procedure of neutron irradiation, transportation for measurement, measurement, and transportation for irradiation; the time sequence of $50,5,150$, and $5 \mathrm{sec}$. was used. The start signal of the one sequence was given manually because of a rule of safety operation. The signal is sent to a digital delay/pulse generator (BNC 575), which controlled the neutron irradiation $(100-\mathrm{Hz}$ neutron generation), transportation, neutron flux monitor, and $\gamma$-ray measurement. The sequence repeated 20 times for one DGS measurement (i.e., approximately $1.2 \mathrm{~h}$ ).

Two uranium oxide standard samples (CBNM-446 and CBNM-031) and one Plutonium alloy sample were interrogated. In the $169-\mathrm{g}$ total Uranium sample, the concentrations of ${ }^{235} \mathrm{U}$ were $4.46 \%$ in CBNM-446 and $0.31 \%$ in CBNM- 031 . The Pu sample was in a coinshaped SUS container of $46 \mathrm{~mm}$ in diameter and $2 \mathrm{~mm}$ in thickness; the ${ }^{239} \mathrm{Pu}$ concentration was $93.7 \%$ of the total 9.4-g Plutonium. Figure 3 shows obtained $\gamma$-ray spectra of CBNM-446 and the Pu samples. Peak assignment was carried out with help of database [12] and Refs. [4-7]. Time evolution of spectra was also obtained from the list data as shown in Fig. 4. This enables us to check the decay time of each peak and to confirm the peak identification. It should be noted that a proper choice of a time period improves signal-to-noise ratio $(\mathrm{S} / \mathrm{N})$ of peaks of a spectrum as seen in Fig. 4.

Many of identified $\gamma$-ray peaks are from parents nuclides with a lifetime in a range from few $10 \mathrm{sec}$. to

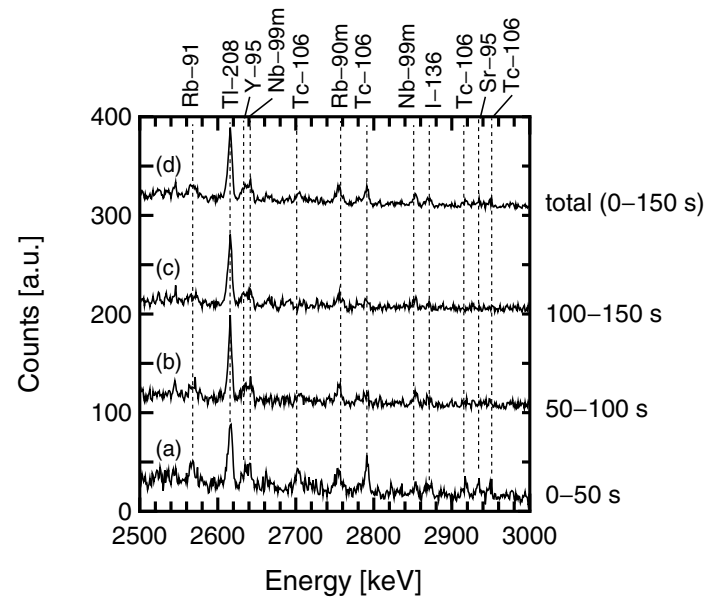

Figure 4. DG spectra of Pu sample obtained each period of time; the spectrum (d) is $1 / 3$ of the sum of the spectra (a-c). Artificial offsets are given to make them easy to see.

several minutes. They are ${ }^{87} \mathrm{Br},{ }^{89,90,90 \mathrm{~m}, 91} \mathrm{Rb},{ }^{95} \mathrm{Y},{ }^{95} \mathrm{Sr}$,

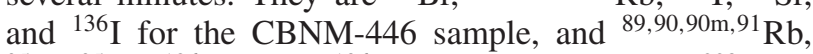
${ }^{95} \mathrm{Y},{ }^{95} \mathrm{Sr},{ }^{106} \mathrm{Tc}$, and ${ }^{136} \mathrm{I}$ for the $\mathrm{Pu}$ sample. ${ }^{208} \mathrm{Tl}$ is contained radioactivity in the samples. Neutron activation products were also observed in CBNM-446. They are ${ }^{27} \mathrm{Al}(\mathrm{n}, \gamma){ }^{28} \mathrm{Al},{ }^{27} \mathrm{Al}(\mathrm{n}, \alpha){ }^{24} \mathrm{Na}$, and ${ }^{16} \mathrm{O}(\mathrm{n}, \mathrm{p}){ }^{16} \mathrm{~N}$; the last two indicative of high-energy neutrons $(\sim 10 \mathrm{MeV})$ interactions. In comparison with the spectrum of $U$ sample in Fig. 3, the Pu sample shows small $\gamma$-ray peaks of ${ }^{90} \mathrm{Rb}$, appearance of those of ${ }^{106} \mathrm{Tc}$, no significant change of those of ${ }^{95} \mathrm{Y}$. However, the spectra are not so simple. In a precise analysis, overlaps of $\gamma$-rays from other nuclides including minor ones should be taken into account.

To analyze an experimental spectrum, a DGS simulation code is under the development. The input parameters are neutron spectrum, sample composition, time sequence of irradiation-transport-observation, and materials around the detectors (neutron filters and $\gamma$-ray shielding). Figure 5 shows a result of the calculation. The identification of each peak is supplied by the code. It roughly reproduced the experimental spectrum. In the future, the code will be incorporate into an analysis according to fitting of an experimental spectrum using Inverse Monte Carlo (IMC) method [13]. 


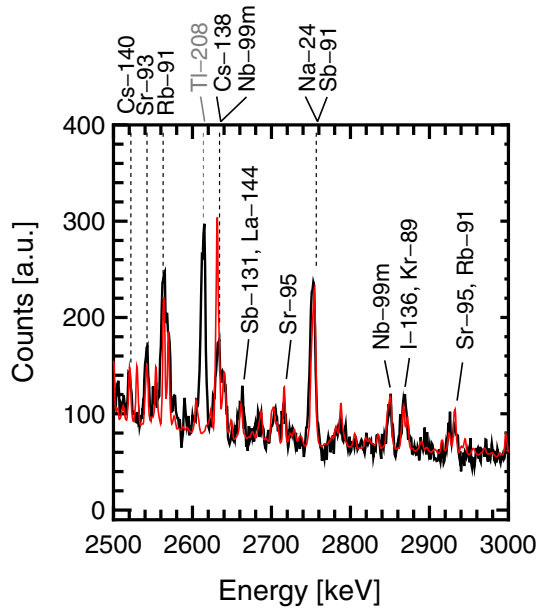

Figure 5. Calculated (red) and experimental (black) spectra of a CBNM-446 sample. To adjust the simulation data to the experimental spectrum, the counts of simulation spectrum are multiplied and a linear base line is added. The peak identification is taken from a program outputs. Decay $\gamma$ ray of ${ }^{208} \mathrm{Tl}$ was not included in this calculation.

\section{Summary}

We are developing DGS technology to determine the ratios of ${ }^{235} \mathrm{U},{ }^{239} \mathrm{Pu}$, and ${ }^{241} \mathrm{Pu}$ in a sample. This NDA method is especially useful for measurement of a sample accompanied with radioactivity, where a conventional passive low-energy $\gamma$-ray spectroscopic method cannot be applied.

Experimental studies with PUNITA have been started at EC-JRC Ispra. Prominent peaks of preliminary obtained $\gamma$-ray spectra of $\mathrm{U}$ and $\mathrm{Pu}$ are assigned. Analyzing list mode data by time stamp may improve $\mathrm{S} / \mathrm{N}$ of a spectrum. Development of an analyzing method of IMC is in progress. In addition to the development of DGS method, nuclear data must be improved in accuracy and availability. Measurements of FY of NMs are in preparation.

This technological research and development was supported by the Japanese government (MEXT). This work was done under the agreement between JAEA and EURATOM in the field of nuclear materials safeguards research and development.

\section{References}

[1] M. Kureta, et al., "JAEA-JRC Collaboration on the Development of Active Neutron NDA Techniques", in the Proceedings of 37th ESARDA Symposium on Safeguards and Nuclear Non-Proliferation (Manchester, UK 19-21 mat 2015), p. 111-120

[2] I. Israelashvili, C. Dubi, H. Ettedgui, A. Ocherashvili, B. Pedersen, A. Beck, E. Roesgen, J.M. Crochmore, T. Ridnik, I. Yaar, Nuclear Instruments and Methods in Physics Research A 785, 14 (2015)

[3] A. Ohzu, M. Koneda, M. Kureta, N. Zaima, Y. Nakatsuka, S. Nakashima, Transactions of the Atomic Energy Society of Japan 15, 115 (2016) (in Japanese)

[4] D. H. Beddingfield and F. E. Cecil, Nucl. Instr. and Meth. in Phys. Res. A 417, 405 (1998)

[5] C.E. Egnatuk, "Identifying short-lived fission products by delayed Gamma-ray emission", Thesis of Univ. Texas, 2009

[6] L.W. Campbell, L.E. Smith, and A.C. Misner, IEEE Transactions on Nucl. Sci. 58, 231 (2011)

[7] B. Ludewigt, V. Mozin, L. Campbell, A.Favalli, A. Hunt, E. Reedy, and H.Seipel, "Delayed GammaRay Spectroscop for Non-Destructive Assay of Nuclear Materials", LLNL-TR-677606 (2015)

[8] H. Postma, and P. Shillebeeckx, in Encyclopedia of Analytical Chemistry, edited by R.A. Meyers (John Wiley \& Sons Ltd., 2009), p. 1

[9] A. Favalli, H.-C. Mehner, J.-M. Crochemore, and B. Pedersen, IEEE Transactions on Nucl. Sci. 56, 1292 (2009)

[10] H. Rennhofer n, J.-M. Crochemore, E. Roesgen, B. Pedersen, Nucl. Instr. Meth. in Phys. Res. A 652, $140(2011)$

[11] X-5 Monte Carlo Team, "MCNP - A General N-Particle Transport Code, Version 5" Volume I: Overview and Theory, LA-UR-03-1987 (2003, updated 2005)

[12] Evaluated Nuclear Structure Data File of National Nuclear Data Center (http://www.nndc.bnl. gov/)

[13] D.C. Rodriguez to be submitted

[14] F.-J. Hambsch, H.-H. Knitter and C. BudtzJorgensen, and J.P. Theobald, Nucl. Phys. A 491, 56 (1989)

[15] W. Mondelaers and P. S hillebeecks, Notiziario Neutroni e Luce di Sincrotrone 11, 9 (2006) 